\title{
Thymic Stromal Lymphopoietin Over-Expressed in Human Atherosclerosis: Potential Role in Th17 Differentiation
}

\author{
Jing Lin ${ }^{\mathrm{a}, \mathrm{d}} \quad$ Wei Chang a,b,d Jiangchuan Dong ${ }^{\mathrm{a}} \quad$ Fengxiao Zhang ${ }^{\mathrm{a}} \quad$ Nilesh Mohabeer $^{\mathrm{a}}$ \\ Kishan Kumar Kushwaha ${ }^{a}$ Lei Wang ${ }^{c}$ Yousu Suc Hongcheng Fang ${ }^{c}$ Dazhu Lia
}

aDepartment of Cardiology, Institute of Cardiovascular Diseases, Union Hospital, Tongji Medical College, Huazhong University of Science and Technology, Wuhan; 'bepartment of Cardiology, Wuhan the first Hospital, Wuhan; 'Department of Cardiology, Shenzhen Sixth People's Hospital (Nanshan Hospital), Huazhong University of Science and Technology, Union Shenzhen Hospital, Shenzhen; djing Lin and Wei Chang contributed equally to this work

\section{Key Words}

TSLP • DCs • Th17 inflammation • Atherosclerosis

\begin{abstract}
Background: Adaptive immunity plays a critical role in atherosclerosis and Thelper 17 (Th17) cells, a new T-cell lineage, are recently reported to be involved in atherosclerosis. However, the mechanism underlying Th17 inflammation in atherosclerosis remains largely unknown. Thymic stromal lymphopoietin (TSLP) is a novel IL-7-like cytokine and mainly responsible for Th2 inflammation in many inflammatory diseases. Methods and Results: Immunohistochemistry showed that TSLP over-expressed in human atherosclerotic lesion and could be induced by oxLDL in human vascular smooth muscle cells (HVSMCs) and human umbilical vein endothelial cells (HUVECs). TSLP, in turn, could activate dendritic cells (DCs) to differentiate Th17 inflammation in naive CD4 ${ }^{+}$T cells. Conclusion: TSLP induced by ox-LDL could promote Th17 immune response in vitro, which may be implicated in Th17 inflammation in atherosclerosis.
\end{abstract}

Copyright (C) 2013 S. Karger AG, Basel

\section{Introduction}

Atherosclerosis is a lipid-driven, chronic inflammatory disease of the vessel wall in which both innate and adaptive immune responses are involved [1]. Immune cells and their mediators directly cause the chronic arterial inflammation that is a hallmark of atherosclerosis. Among these immune cells, $\mathrm{T}$ cells are of fundamentally critical for lesion development because of its important role in innate and adaptive inflammation. 
Based on specific CD3 antibody staining, numerous amounts of $\mathrm{CD}^{+} \mathrm{T}$ cells are found in atherosclerotic plaques. Most of these are in activated condition, as evidenced by their proliferation, secretion of cytokines, and expressions of adhesion molecules as well as other surface molecules. CD4+ $\mathrm{T}$ cells in lesion thus grouped as Th1, Th2, and Th17 cell subsets. While Th1 and Th17 inflammatory response have been confirmed to be pro-atherosclerosis, Th2 inflammation seems to be little impact on atherosclerosis $[1,2]$. Thus, to elucidate how to regulate and influence the specific immune cells and general immune paradigms, such as Th1, Th2 and Th17 in atherosclerosis is fundamental important.

Thymic stromal lymphopoietin (TSLP) is a novel IL-7-like cytokine and generally produced by epithelial cells, fibroblasts, mast cells, and keratinocytes [3]. Signaling of cells by TSLP requires IL-7 receptor- $\alpha$ and a distinctive receptor subunit, the TSLP receptor (TSLPR), which is expressed by dendritic cells (DCs), monocytes, preactivated T cells, natural killer cells, and mast cells [4]. In humans, TSLP/TSLPR could stimulate DCs, with up-regulated expression of HLA-DR, CD40, CD80, CD86, OX40L, and CD83 and production of chemokines, including thymus and activation-regulated chemokine (TARC), macrophage-derived chemokine (MDC), IL-8 and IL-15 [3, 5]. Numerous studies had confirmed the important role of TSLP-activated DCs in T cell lineage choice. TSLP-activated DCs their turn stimulate naive CD4 $\mathrm{T}$ cells to proliferate, polarize and induce central memory $\mathrm{T}$ cells, which retain the capacity to differentiate into different cell subsets. The ability of TSLP-activated DCs on Th2 differentiation has been well studied in several autoimmune disorders because of its effects on Th2 cytokine-secreting [6]. TSLP activated DCs, which induces the production of Th2-attracting chemokines such as TARC and MDC, which then prime development of T cells producing Th2 cytokines. In atopic dermatitis, TSLP-driven Th2 cytokine is over-expressed in acute and chronic skin lesions, and in allergic asthma, TSLP is increased in the airways and correlates with Th2 activity and disease severity. However, there is some evidence that TSLP may also contribute to Th17 cytokine-dependent inflammation [7-9]. Hartgring et al. reported whereas administration of TSLP significantly exacerbated collagen-induced arthritis and the joint damage which characterized by increased Th17 activation, TSLPR-/- in arthritis mice had diminished concentrations of Th17-related pro-inflammatory mediators, including IL-17, IL-1 $\beta$, IL-6, and matrix metalloproteinase 9 [8]. Our previous study found that TSLP could be inductive by angiotensin II and promoted Th17 inflammation in rat vascular smooth muscle cells, while Lee et al. found TSLP could be inductive by Hepatitis C virus and promoted Th7 responses in infected hepatocytes $[9,10]$.

These above-mentioned data collectively indicated an important role of TSLP in T cells adaptive inflammation. However, the underlying mechanism under TSLP and Th17 inflammation still remains largely unknown. Now, TSLP mediated inflammation has been confirmed in many inflammatory diseases, such as asthma, atopic dermatitis and arthritis [6, $8,11]$. However, the functional properties of TSLP in atherosclerosis remain unknown. With consideration of the central role of TSLP and DCs in driving T lineage choice, the possible role of TSLP in atherosclerosis was assessed with in vitro experiments.

\section{Materials and Methods}

\section{Human tissue samples}

This study was approved by the Ethics Committee of Tongji Medical College, Huazhong University of Science and Technology, China. All patients gave written informed consent and research was conformed to the guidelines of the declaration of Helsinki and its amendments. Arterial wall specimens containing atherosclerotic plaques (carotid arteries) $(n=6)$ were obtained from patients who underwent carotid endarterectomy in the Department of Vascular Surgery, Union Hospital. Normal vessels ( $n=4)$ without atherosclerotic disease were used as reference material. After surgery, tissues were halved at the site of the maximum artery diameter. One half was fixed in formalin and embedded in paraffin for histology; the other half was immediately frozen at $-80^{\circ} \mathrm{C}$ for subsequent RT-PCR analysis. The baseline characteristics of the patients are summarized in Table1. 
Lin et al.: A Potential Role of Thymic Stromal Lymphopoietin in Th17 Differentiation in Atherosclerosis

\begin{tabular}{lr}
\hline \multicolumn{2}{c}{ Baseline characteristics of the patients } \\
\hline Patients, n & 10 \\
Age, y & $54 \pm 17$ \\
Male/sex, \% & $60 \%$ \\
Diabetes mellitus, \% & $30 \%$ \\
Systemic hypertension, \% & $50 \%$ \\
Hyperlipoproteinemia, \% & $40 \%$ \\
Smoking, \% & $50 \%$ \\
History of myocardial infarction, \% & $20 \%$ \\
\hline
\end{tabular}

Table 1. Baseline characteristics of the patients

\section{Immunohistochemistry analysis}

For tissues, sections were routinely stained with haematoxylin-eosin and then stained with antihuman TSLP Ab (Santa Cruz, USA). The reaction products were visualized by treating the slide with 3, 30-diaminobenzidine tetra hydrochloride (DAB Liquid System, Dako) and counterstained with haematoxylin. For control, the primary antibody was replaced by PBS or by non-specific human IgG. Immunohistochemistry for cells (IHC) was performed using anti-TSLP (Santa Cruz, USA) or anti-CD11 Ab (Santa Cruz, USA, and with secondary antibodies with peroxidase or with secondary antibodies labeled with PE (eBioscience, USA). For control, primary antibodies were replaced with non-specific immunoglobulins. For quantitative analysis of immunohistochemistry, images were visualized and analyzed with HMIAS Series Color Medical Image Analyze System (Champion Image Ltd., China) or visualized with a fluorescent microscope (Olympus Microscope BX-51, Japan) and quantified using designed software.

\section{Isolation and culture of HUVECS}

Human umbilical vein endothelial cells (HUVECs) were isolated from human umbilical veins that were cannulated, perfused with Hanks' solution to remove blood, and then incubated with $1 \%$ collagenase for 15 minutes at $37^{\circ} \mathrm{C}$. After removal of collagenase (Sigma, USA), cells were cultured in Ml99 medium (Gibco, USA) supplemented with 20\% foetal calf serum (Gibco, USA), $100 \mathrm{ug} / \mathrm{ml}$ heparin (Sigma, USA), 50 $\mathrm{ug} / \mathrm{ml}$ endothelial cell growth supplement (Gibco, USA), 25 mM Hepes buffer, 2 mM L-glutamine, 100 U/ $\mathrm{ml}$ penicillin, and $100 \mathrm{ug} / \mathrm{ml}$ streptomycin, and grown at $37^{\circ} \mathrm{C}$ on tissue culture plates coated with $0.1 \%$ gelatin. Cells were passaged at confluence by splitting1:4 and were used within the first eight passages. Use of human umbilical veins from normal donors was approved by the Ethics Committee of Tongji Medical College, Huazhong University of Science and Technology.

After cultured with different concentrations of ox-LDL $(0 \mathrm{mg} / \mathrm{L}, 25 \mathrm{mg} / \mathrm{L}, 50 \mathrm{mg} / \mathrm{L}, 100 \mathrm{mg} / \mathrm{L})$ for $12 \mathrm{~h}$, HUVECs were harvested and supernatants were collected for further experiments.

\section{Isolation and culture of HVSMCS}

Human vascular smooth muscle cells (HVSMCs) were obtained by enzymatic dissociation of the superficial femoral artery. The arterial fragments were cleaned free of the adventitia layer then the endothelium was removed by gently rubbing the lumen of the vessel. The fragments were cut into small pieces and digested by incubation for 90 min in Dulbecco's Modified Eagle's Medium (DMEM; Sigma USA) containing $0.1 \%$ BSA and $4 \mathrm{mg} / \mathrm{ml}$ collagenase (type II, Sigma, USA). The resulting cell suspension was washed twice with fresh DMEM and then plated onto tissue culture plates in DMEM containing $10 \%$ foetal calf serum, $100 \mathrm{U} / \mathrm{ml}$ penicillin and $100 \mathrm{ug} / \mathrm{ml}$ streptomycin. Cell characterization was performed based on both cell morphology and indirect immunohistochemistry staining of $\alpha$-smooth muscle actin. Cells were passaged at confluence by splitting1:2 and were used within the first eight passages.

After cultured with different concentrations of ox-LDL $(0 \mathrm{mg} / \mathrm{L}, 25 \mathrm{mg} / \mathrm{L}, 50 \mathrm{mg} / \mathrm{L}, 100 \mathrm{mg} / \mathrm{L})$ for $12 \mathrm{~h}, \mathrm{HVSMCs}$ were harvested and supernatants were collected for further experiments.

Isolation and culture of DCs and CD4+ T cells

Isolation of human peripheral blood mononuclear cells (PBMCs) which obtained from normal volunteers was performed using Ficoll-Hypaque density gradient centrifugation following the manufacturer's protocol (Human lymphocyte separation medium from Chinese Academy of Medical Sciences Institute of Biomedical 
Engineering, China.) CD4+T cells were isolated by magnetic affinity cell sorting (MACS) using the CD4+beads kit (Miltenyi Biotec, Germany).

Purified CD14+ monocytes isolated from PBMCs by MACS were cultured with recombinant human IL-4 (rhIL-4; $100 \mathrm{U} / \mathrm{mL}$, PeproTech, USA) and recombinant human granulocyte-macrophage colony-stimulating factor (100 U/mL, PeproTech, USA) for 7 days. The floating cells with short dendrite were further isolated using CD11c bead-based MACS, which were named peripheral blood DCs with a purity of more than $95 \%$.

\section{Cell co-culture system}

HUVECs or HVSMCs were cultured with ox-LDL $(50 \mu \mathrm{g} / \mathrm{mL})$ for $24 \mathrm{~h}$, and then supernatant from HUVECs (supernatant (E)) or HVSMCs (supernatant (M)) were collected for further stimulation. DCs were cultured with theses supernatants for $24 \mathrm{~h}$, washed 3 times, and then co-cultured with $\mathrm{CD} 4^{+} \mathrm{T}$ cells in the transwell technique according to the protocol of Qiao et al. with some modifications. After 5 days, $\mathrm{CD} 4^{+} \mathrm{T}$ cells were stimulated with anti-CD3 $(5 \mu \mathrm{g} / \mathrm{mL}$, OKT3, eBioscience, USA $)$ and anti-CD28 $(1 \mu \mathrm{g} / \mathrm{mL}$, eBioscience, USA $)$ and cultured for $24 \mathrm{~h}$. In some experiments, anti-TSLP neutralizing antibody (1ng/mL, eBioscience, USA) or anti-nonblocking antibody were added at the start of co-culture system.

\section{Inhibition experiments}

DCs and $\mathrm{CD} 4^{+} \mathrm{T}$ cells were cultured as described above. $40 \mu \mathrm{mol} / \mathrm{l}$ STAT3 pathway inhibitor D4071 (Sigma, USA) were added to the culture supernatants at the start of co-culture and DMSO was used as a control.

Preparation of LDL and copper-oxidized LDL

Blood for lipoprotein isolation was collected in EDTA $(1 \mathrm{mg} / \mathrm{mL})$ from normal lipidemia donors after 12 hours of fasting. LDL (density=1.03 to $1.063 \mathrm{~g} / \mathrm{L}$ ) was isolated from the plasma after density adjustment with $\mathrm{KBr}$, by preparative ultracentrifugation at $50000 \mathrm{rpm} / \mathrm{min}$ for 22 hours, using type 50 rotor. They were dialyzed against phosphate buffered saline (PBS) containing 0.3mM EDTA, sterilized by filtration through a $0.22-\mathrm{mm}$ filter, and stored under nitrogen gas at $4^{\circ} \mathrm{C}$. Protein content was determined by the method of Lowry et al. Copper oxidation of LDL was performed by incubation of post-dialyzed LDL (1mg of protein $/ \mathrm{ml}$ in EDTA-free PBS) with copper sulfate $(10 \mathrm{mM})$ for 24 hours at $37^{\circ} \mathrm{C}$. Lipoprotein oxidation was confirmed by analysis of thiobarbituric acid-reactive substances [12].

\section{Cytokines assay}

Cultured supernatants or patients' blood serum were collected and kept frozen at $-80^{\circ} \mathrm{C}$ until the cytokine levels (TSLP, IFN- $\gamma$, IL-22, IL-23, IL-6, TNF- $\alpha$, IL-10, TRAC, and MDC) were determined by ELISA assays according to the manufacturer's instructions. All commercial kits were purchased from R\&D Systems (USA). Each sample was tested in triplicate.

\section{RT-PCR analysis}

The level of TSLP and TSLPR mRNA expression was analyzed by real-time reverse transcriptionpolymerase chain reaction (Real-time RT-PCR). Cells were harvested from cells or tissues, and total RNA was isolated using Trizol reagent (Invitrogen, USA) according to the manufacturer's recommendations. RNA concentration and its purity were measured by a spectrophotometer. One microgram of total RNA was reversely transcripted into cDNA using RNA PCR Kit (Takara Biotechnology, Japan) and the cDNA was used as a template. The real-time PCR was carried out with Applied Biosystems Step One Real-Time PCR System (Applied Biosystem, USA) according to the manufacturer's protocol. A housekeeping genes GAPDH was used as an internal control for the PCRs. The sequences of the primers were presented at Table2. The PCR reaction mixture was prepared with the SYBR Green I (Takara Biotechnology, Japan), cDNA, and the primers following the manufacturer's protocol. The amount of gene expression was then calculated as the difference $(\Delta \mathrm{Ct})$ between the $\mathrm{Ct}$ value of target gene and the $\mathrm{Ct}$ value of GAPDH. Fold changes in target gene mRNA were determined by the formula $2^{-\Delta \Delta c t}$.

\section{Western Blot analysis}

Equal protein amounts were loaded onto SDS-PAGE gels. After running gels, proteins were transferred onto nitrocellulose membranes. Membranes were blocked in 5\% nonfat milk and primary antibody 
Lin et al.: A Potential Role of Thymic Stromal Lymphopoietin in Th17 Differentiation in Atherosclerosis

\begin{tabular}{lll}
\hline Primer & \multicolumn{1}{c}{ Forward } & \multicolumn{1}{c}{ Reverse } \\
\hline TSLP & CGAGCA AATCGAGGACTGTGA & GCAGTCGTCATTGAGCGCTTC \\
IL-4 & CCACGGACACAAGTGCGATA & CCCTGCAGAAGGTTTCCTTCT \\
IL-13 & GAGTGTGTTTGTCACCGTTG & TACTCGTTGGTCGAGAGCTG \\
IL-6 & AACCTGAACCTTCCAAAGATGG & TCTGGCTTGTTCCTCACTACT \\
IL-23 & TTCTCTGCTCCCTGATAGCC & CCTCAGGCTGCAGGAGTT \\
IL-1 $\beta$ & TTACAGTGGCAATGAGGATGAC & GTCGGAGATTCGTAGCTGGAT \\
IL-17 & TCATCCATCCCCAGTTGATT & GACCAGGATCTCTTGCTGGA \\
IL-22 & TGCAGGCTTGACAAGTCCAA & CTAGCCTCCTTAGCCAGCATGA \\
TNF- $\alpha$ & AACCTGAACCTTCCAAAGATGG & TCTGGCTTGTTCCTCACTACT \\
GADPH & ACCCACTCCTCCACCTTTG & CTCTTGTGCTCTTGCTGGG \\
\hline
\end{tabular}

Table 2. Primers

incubations were performed with $3 \%$ BSA (overnight at $4^{\circ} \mathrm{C}$ ). Antibodies used were anti-phospho-STAT3 (Santa Cruz, USA), and anti- $\beta$ actin (Santa Cruz, USA). Then membranes were incubated with peroxidaseconjugated secondary antibodies at room temperature for 2 hours. Specific band was detected with chemiluminescence assay (ECL detection reagents, Pierce) and recorded on x-ray film. Designed software was used to quantify the intensities of bands.

\section{Flow cytometric analysis}

HUVECs or HVSMCs were cultured with ox-LDL (50 $\mathrm{gg} / \mathrm{mL})$ for $24 \mathrm{~h}$, and then supernatant from HUVECs (supernatant (E)) or HVSMCs (supernatant (M)) were cultured with DCs for $24 \mathrm{~h}$. After $24 \mathrm{~h}$ of co-culture, DCs washed 3 times, and then co-cultured with CD4 ${ }^{+} \mathrm{T}$ cells for 5 days in the transwell system. Monensin was used to block the intracellular transport mechanisms, thereby leading to an accumulation of cytokines in the cells. In some experiments, anti-non-blocking antibody or anti-TSLP neutralizing antibody were added when ox-LDL were cultured with DCs. After incubation, cells were aliquoted into tubes and washed in PBS, and then resuspended to a density of $10^{6} \mathrm{cells} / \mathrm{mL}$. Cells were then incubated with FITC anti-human-CD4 at $4{ }^{\circ} \mathrm{C}$ for $20 \mathrm{~min}$ for cell surface staining. After surface staining, fixation and permeabilization, cells were stained with phycoerythrin-Cy5 (PE/Cy5) anti-human-IL-17 for Th17 detection (eBioscience, CA). Isotype controls were given to enable correct compensation and confirm antibody specificity. Stained cells were analyzed by flow cytometric analysis using a FACS can cytometer equipped with CellQuest software (BD Bioscience Pharminggen).

\section{Statistical Analyses}

Results are shown as mean \pm SD of at least three independent experiments. The significance of differences was determined using ANOVA followed by Student-Newmann-Keuls multiple comparison tests. $P$ values of $<0.05$ were considered statistically significant. All statistical analyses were performed with SPSS 13.0 (SPSS Inc., Chicago, IL).

\section{Results}

TSLP over-expressed in human atherosclerotic artery specimens

Figure 1 showed a marked expression of TSLP protein in a human carotid endarterectomy specimen, most notably in the smooth muscle cells-rich layer (Fig. 1B and C). The TSLP protein was also found not only in within endothelial-rich layer of intimae (Fig. 1D). Because the localization of TSLP in atherosclerotic tissue was solely based on morphological criteria, TSLP mRNA in human normal vessels and atherosclerotic artery specimens was also detected in our study. Real-time PCR showed a 2.8 \pm 1 .1-fold up-regulation of TSLP mRNA in human atherosclerotic specimens $(n=6)$ as compared with non-atherosclerotic arteries $(\mathrm{n}=4)$ (Fig. 1E).

Immunohistochemical staining showed that TSLP in atherosclerotic lesion was mainly co-located within CD11 positive reaction (Fig. 2), which was consistent with previous studies that DCs are the major target of TSLP[3]. 

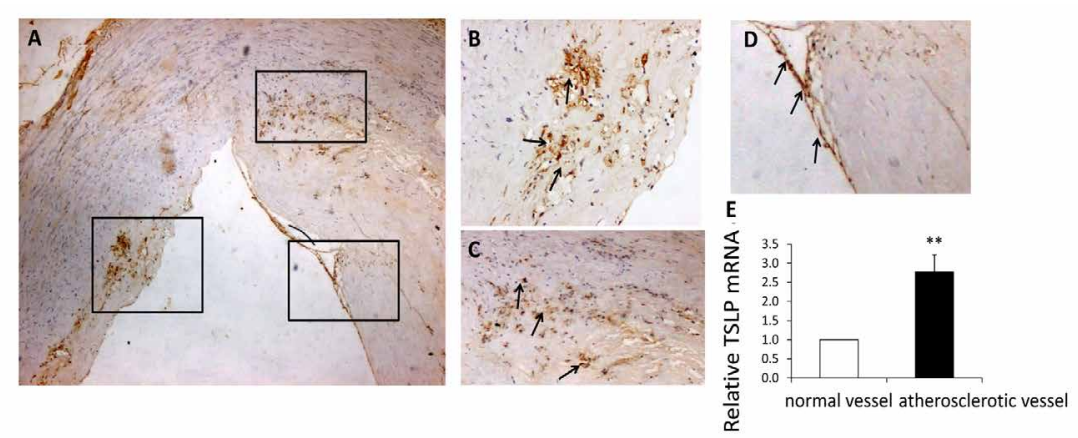

Fig. 1. Immunohistochemical detection of TSLP in atherosclerotic artery specimens. (A) Low-power photomicrograph of an human atherosclerotic specimens stained for TSLP (magnification $\times 40$ ).(B)-(D), High-power photomicrograph of upper boxed area in A showing strong TSLP staining in the atherosclerotic tissue (arrows)(magnification x100). (E) RT-PCR analysis of TSLP mRNA in normal vessel specimens $(n=4)$ and atherosclerotic artery specimens $(n=6)$. Data are presented as mean \pm SEM of at least three independent experiments. ${ }^{*}$ indicated vs normal vessel specimens, ${ }^{* *} \mathrm{p}<0.01$.

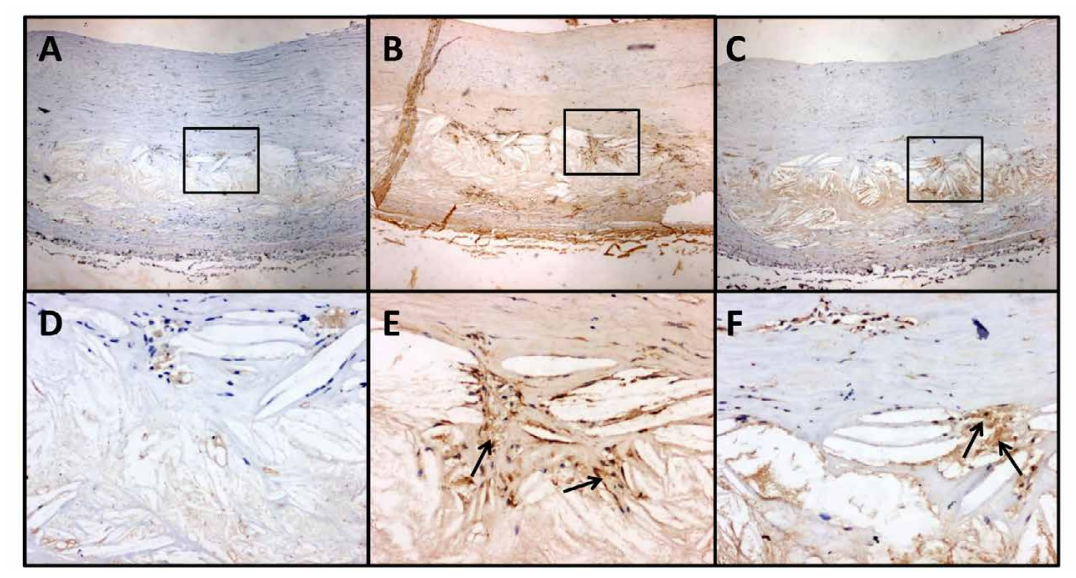

Fig. 2. Immunohisto-chemical staining of TSLP and CD11+ in atherosclerotic artery specimens. Consecutive sections of human carotid endarterectomy specimen (A to C) were stained with nonspecific human IgG antibodies (isotype control) (A); TSLP (B); and CD11 (C) (magnification x40). Highmagnification (x200) views of lesion area specified with rectangular boxes in $A$ to $C$ are shown in $D$ to $F$, respectively.

Fig. 3. mRNA of Th17 related cytokines increased in normal vessel and atherosclerotic artery specimens. RT-PCR analysis of Th17 related cytokines (IL-6, IL23, IL-1 $\beta$, IL-17, IL-22 and TNF- $\alpha$ ) mRNA in normal vessel specimens $(n=4)$ and atherosclerotic artery specimens $(n=6)$. Data are presented as mean \pm SEM of at least three independent experiments.

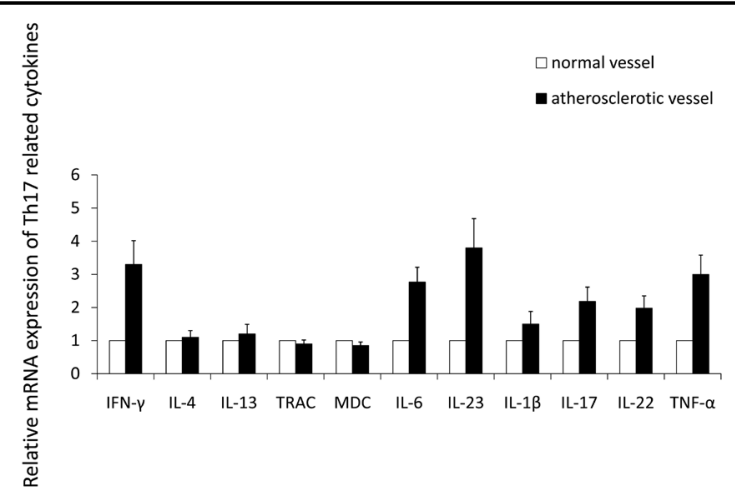

mRNA of Th17 related cytokines increased in normal vessel and atherosclerotic artery specimens

Numbers of studies had reported that Th17 related cytokines was up-regulated in atherosclerosis $[13,14]$. Consistent with these results, our study found that mRNA of 


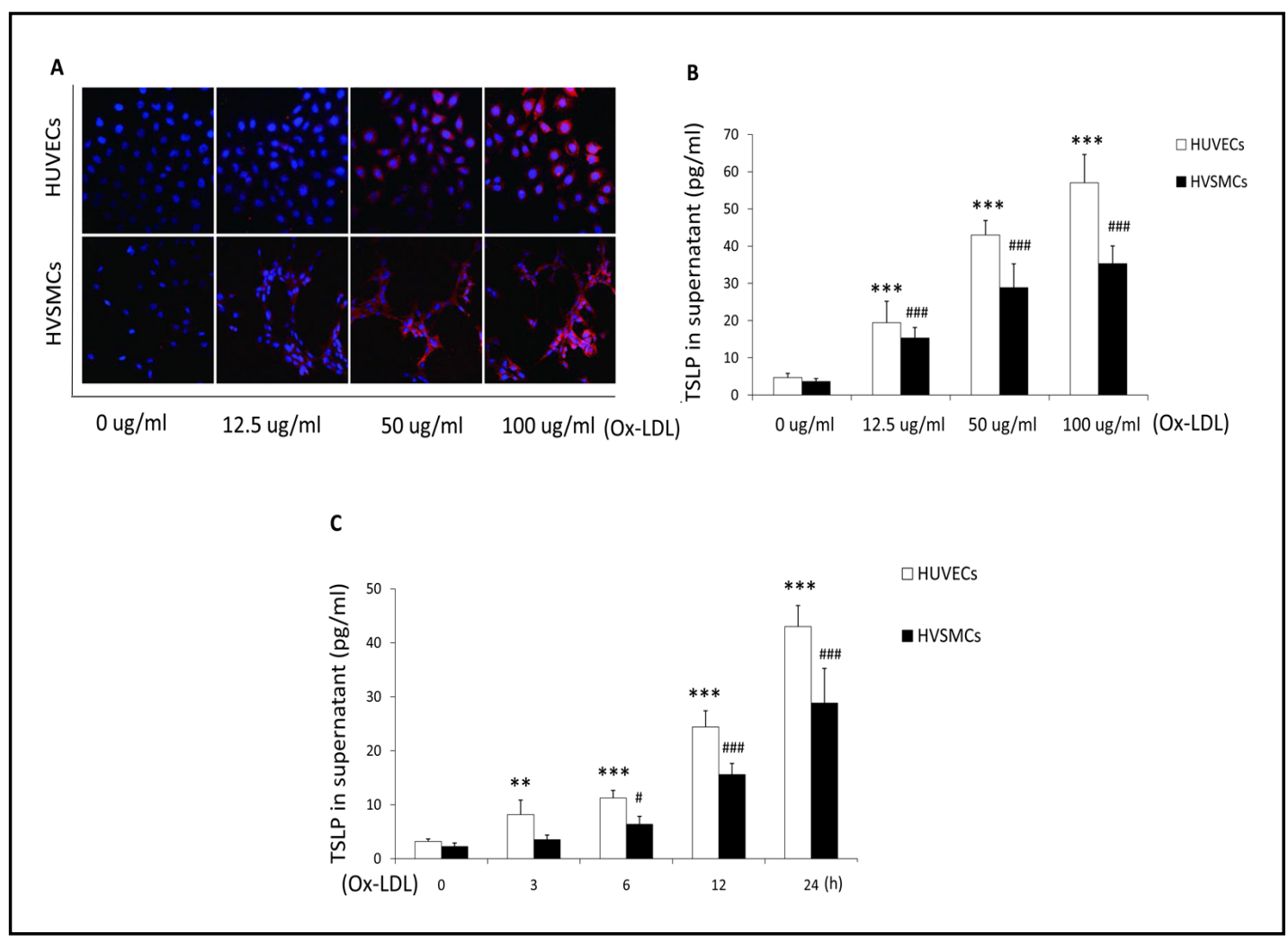

Fig. 4. Ox-LDL induced TSLP in HUVECs and HVSMCs. (A) HUVECs or HVSMCs were cultured in the presence of ox-LDL at indicated concentration for $24 \mathrm{~h}$, then TSLP (red) in HUVECs or HVSMCs were detected by IHC. (B) HUVECs or HVSMCs were cultured in the presence of ox-LDL at indicated concentration for $24 \mathrm{~h}$, then TSLP (red) in HUVECs or HVSMCs were detected by ELISA. (C) HUVECs or HVSMCs were cultured in the presence of ox-LDL (50ug/ml) for indicated time, then TSLP in HUVECs or HVSMCs were detected by ELISA. * indicated vs untreated HUVECs, ${ }^{* *} \mathrm{p}<0.01$; ${ }^{* * *} \mathrm{p}<0.001$. \# indicated vs untreated HVSMCs, \#p $<0.05$; $\# \# \#$ p $<0.001$. Data are presented as mean \pm SEM of at least three independent experiments.

Th17 related cytokines (IL-6, IL-23, IL-1 $\beta$, IL-17, IL-22 and TNF- $\alpha$ ) were increased in atherosclerotic artery specimens than which in normal vessels (Fig. 3).

OX-LDL induced TSLP expression in HVSMCs and HUVECS

To unravel the mechanism of TSLP expression in atherosclerotic plaques, ox-LDL, the major existence for atherosclerogensis, was selected as stimuli. Because the localization of TSLP in SMCs and endothelium previously was solely based on morphological criteria, HVSMCs as well as HUVECs were used stimulation with ox-LDL in vitro. Immunohistochemical staining of TSLP in HVSMCs and HUVECs was performed as described in Methods. Figure 4A showed that whereas no staining of TSLP was detected within HVSMCs and HUVECs in absence of ox-LDL, strong positive staining of TSLP (red) was observed in HVSMCs and HUVECs after exposure to ox-LDL (50ug/ml or $100 \mathrm{ug} / \mathrm{ml}$ ) for $24 \mathrm{~h}$.

To further confirm TSLP production in these cells, the level of TSLP protein in cell supernatants was measured by ELISA. Our results showed that ox-LDL induced an upregulated TSLP release in a concentration and time-dependent manner. At 24-hour time point, a statistically significant increase of TSLP was found at $12.5 \mu \mathrm{g} / \mathrm{mL}$ ox-LDL in both HUVECs and HVSMCs (p< 0.001) (Fig. 4B). Upon stimulation with ox-LDL (50 $\mu \mathrm{g} / \mathrm{mL})$, a statistically significant increase of TSLP was observed in HUVECs at 3-hour time point $(\mathrm{p}<0.01)$ while at 6-hour time point $(\mathrm{p}<0.05)$ in HVSMCs (Fig. 4C). 

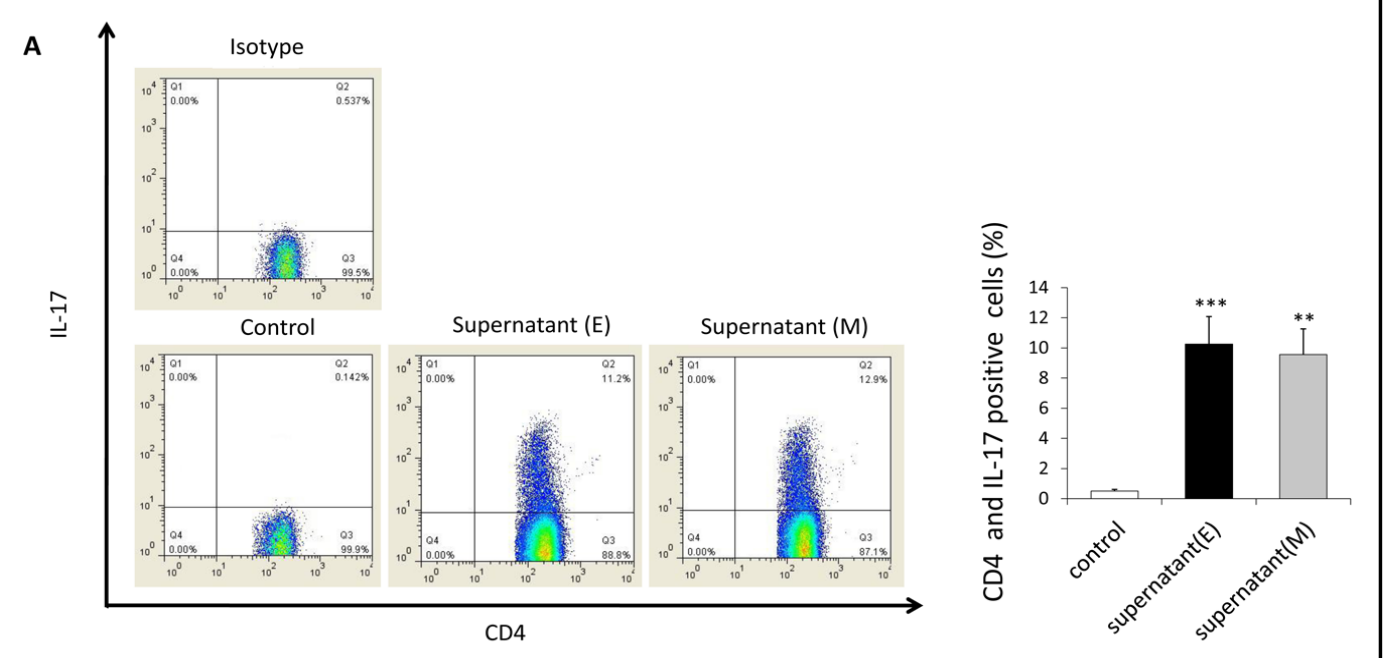

B

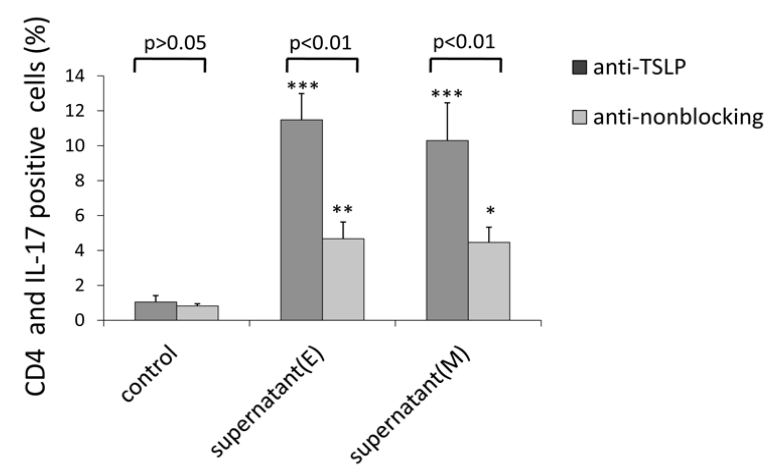

Fig. 5. TSLP-DCs Differentiated Th17 cells in CD4+ cells. HUVECs or HVSMCs were cultured with ox-LDL $(50 \mu \mathrm{g} / \mathrm{mL}$ ) for $24 \mathrm{~h}$, and then supernatant from HUVECs (supernatant (E)) or HVSMCs (supernatant (M)) were cultured with DCs for $24 \mathrm{~h}$. After incubation, DCs washed 3 times, and then co-cultured with CD4 ${ }^{+}$ $\mathrm{T}$ cells for 5 days in the transwell system. In some experiments, anti-non-blocking antibody or anti-TSLP neutralizing antibody were added when ox-LDL were cultured with DCs. Details were described in methods. DCs were cultured with PBS and then cultured with CD4+ $\mathrm{T}$ cells as control. (A) CD4+ $\mathrm{T}$ cells were sequentially stained with FITC-conjugated anti-CD4 antibody and PE-conjugated anti-IL-17 antibody, and analyzed by flow cytometry in gated CD4+IL-17+ cells. Frequencies of CD4+IL-17+ cells were analyzed statistically by SPSS 13.0. * indicated vs control, ${ }^{* *} \mathrm{p}<0.01 ;{ }^{* * *} \mathrm{p}<0.001$. (B) Frequencies of CD4+IL-17+ cells were analyzed statistically by SPSS 13.0. * indicated vs control cells cultured with anti-non-blocking antibody, ${ }^{* *} \mathrm{p}<0.01$; $* * * \mathrm{p}<0.001$. Data are presented as mean \pm SEM of at least three independent experiments.

\section{Supernatant TSLP induced by ox-LDL differentiated Th17 cells via dendritic cells}

TSLP has been vastly studied in human atopic disorders because of its effects on differentiating of Th2 T cells. However, TSLP may also contribute to Th17 cytokines dependent inflammation. To examine the effect of ox-LDL-induced TSLP on CD4+ cells differentiation, supernatant of ox-LDL $(50 \mathrm{ug} / \mathrm{ml})$ treated HVSMCs (supernatant(M))or HUVECs (supernatant(E)) incubated with DCs for $24 \mathrm{~h}$, then DCs were co-cultured with CD4+ cells for additional $24 \mathrm{~h}$ and FACS was performed to measure the proportion of Th17+ cells. Figure 5 showed compared with untreated DCs (control), supernatant TSLP treated DCs significantly increased the percentage of Th17 in CD4+ cells ((supernatant (E):0.87 $\pm 0.22 \%$ versus $10.14 \pm 2.38 \%, p<0.01$; (supernatant $(\mathrm{M})$ : $0.87 \pm 0.22 \%$ versus $9.88 \pm 2.16 \%, \mathrm{p}<0.01$ ). Our results also showed that adding anti-TSLP neutralizing antibody in the start of co-culture system blocked Th17 differentiation in CD4+ cells (supernatant (E): 10.14 $\pm 2.38 \%$ versus $4.26 \pm 1.82 \%$, $\mathrm{p}<0.01$; supernatant $(\mathrm{M})$ : $9.88 \pm 2.16 \%$ versus $3.42 \pm 1.15 \%, \mathrm{p}<0.01$ ), indicating 


\begin{tabular}{|c|c|c|}
\hline Cellular Physiology & Cell Physiol Biochem 2013;31:305-318 & \\
\hline and Biochemistry & $\begin{array}{l}\text { Dol: } 10.1159 / 000343369 \\
\text { Published online; }\end{array}$ & $\begin{array}{l}\text { o } 2013 \text { S. Karger AG, Basel } \\
\text { wwwwkaraercom/cob }\end{array}$ \\
\hline
\end{tabular}

Fig. 6. TSLP-DCs induced Th17 cytokines in $\mathrm{CD}^{+} \mathrm{T}$ cells. Cells were cultured as described in legend 4. Supernatant in the cultures were collected and tested for IFN- $\gamma$, IL-4, IL-13, TNF- $\alpha$, IL-17 and IL-22 by ELISA. (A)* indicated vs $\quad$ control, ${ }^{*} \mathrm{p}<0.05$; ${ }^{* *} \mathrm{p}<0.01 ; \quad{ }^{* * *} \mathrm{p}<0.001$.

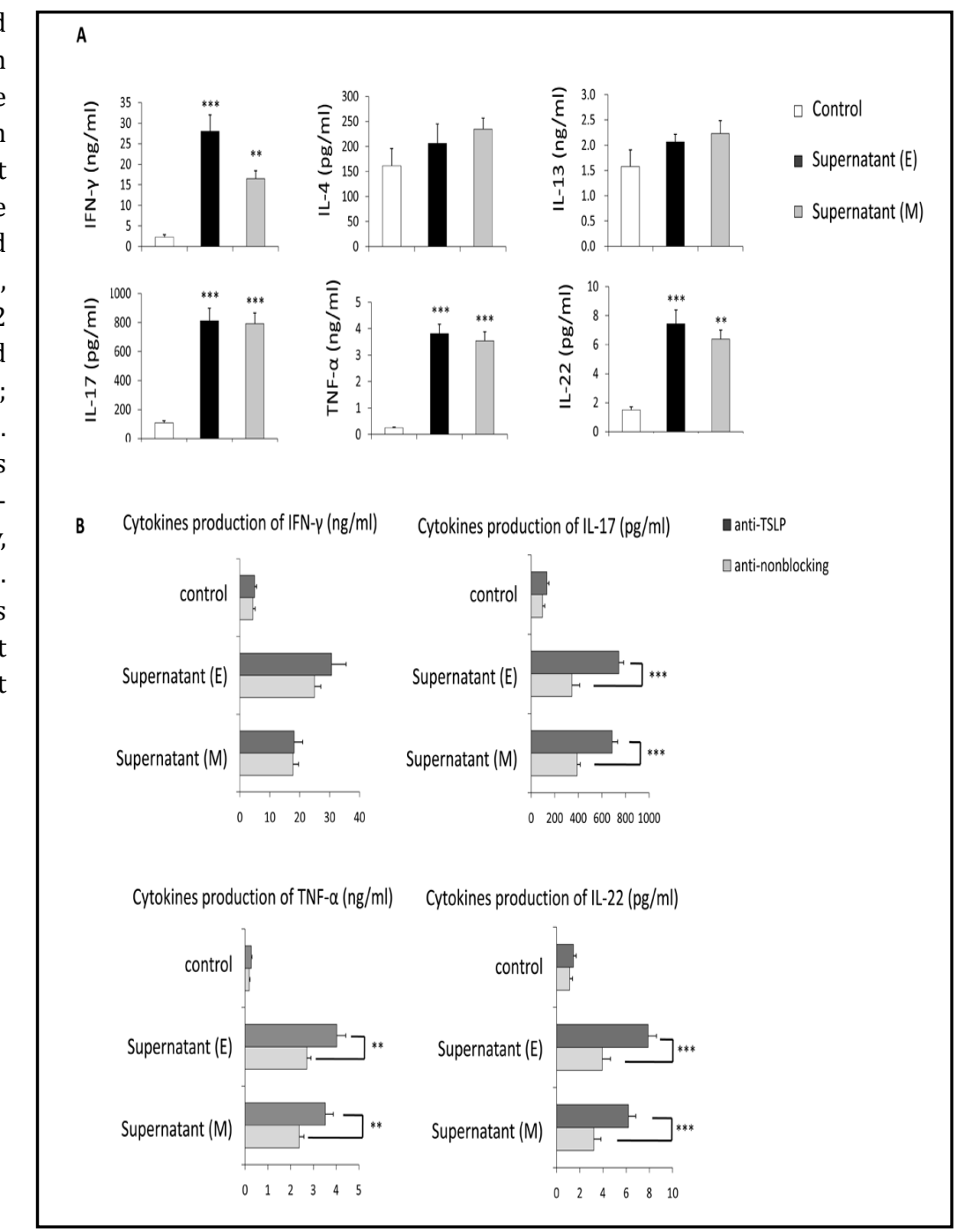
$(\mathrm{B})^{*}$ indicated vs DCs cultured with antinon-blocking antibody, ${ }^{* *} \mathrm{p}<0.01 ; \quad{ }^{* * *} \mathrm{p}<0.001$. Data are presented as mean \pm SEM of at least three independent experiments.

TSLP induced by ox-LDL in the supernatant was mainly responsible for DCs induced Th17 differentiation.

TSLP-DCs stimulated the production of Th17 cytokines in $C D 4^{+}$T cells

The inflammatory cytokines and mediators in the supernatant TSLP-DCs primed T cells were also determined in our study. In partly consistent with previous studies, TSLP-DCs in our study induced a typical characteristic of Th17 inflammatory response, as shown by the up-regulated cytokines of IL-17, IL-22 and TNF- $\alpha$ (Fig. 6A).TSLP-DCs failed to induce production of IL-13 and IL-4 from primed T cells (Fig. 6A). Furthermore, Th1 cytokine, IFN- $\gamma$ was also up-regulated in primed $\mathrm{CD} 4^{+} \mathrm{T}$ cells (Fig. 6A).

Next we examine the role of TSLP in supernatant-DCs induced cytokines production. Our results found that neutralizing antibody of TSLP could largely block supernatant-DCs induced production of IL-17, IL-22 and TNF- $\alpha$ (Fig. 6B), indicating that it was TSLP in the supernatant to stimulate DCs to response Th17 inflammatory activity. In contrast, anti-TSLP neutralizing antibody had no effect on IFN- $\gamma$ production.

\section{Cytokine profiles of TSLP induced DCs}

Because cytokine and chemokine production by activated DCs plays a critical role in the differentiation of primed T cells, cytokine and chemokine production in supernatant of 
Fig. 7. TSLP induced cytokines and chemikines in DCs. HVSMCs were cultured with ox-LDL $(50 \mu \mathrm{g} / \mathrm{mL})$ for $24 \mathrm{~h}$, and then supernatant was cultured with DCs for 24 h. After incubation, DCs washed 3 times and cultured for additional $24 \mathrm{~h}$ and supernatant were collected and tested for TRAC, MDC, IL-1 $\beta$, IL- 6 and IL-23 by ELISA. DCs cultured with PBS were as control. In some experiments, anti-nonblocking antibody or antiTSLP neutralizing antibody were added when ox-LDL were cultured with DCs. $(\mathrm{A})^{*}$ indicated vs controls, ${ }^{* *} \mathrm{p}<0.01 ; \quad{ }^{* * *} \mathrm{p}<0.001$. (B)* indicated vs DCs cultured with anti-non-blocking antibody, least three independent experiments.

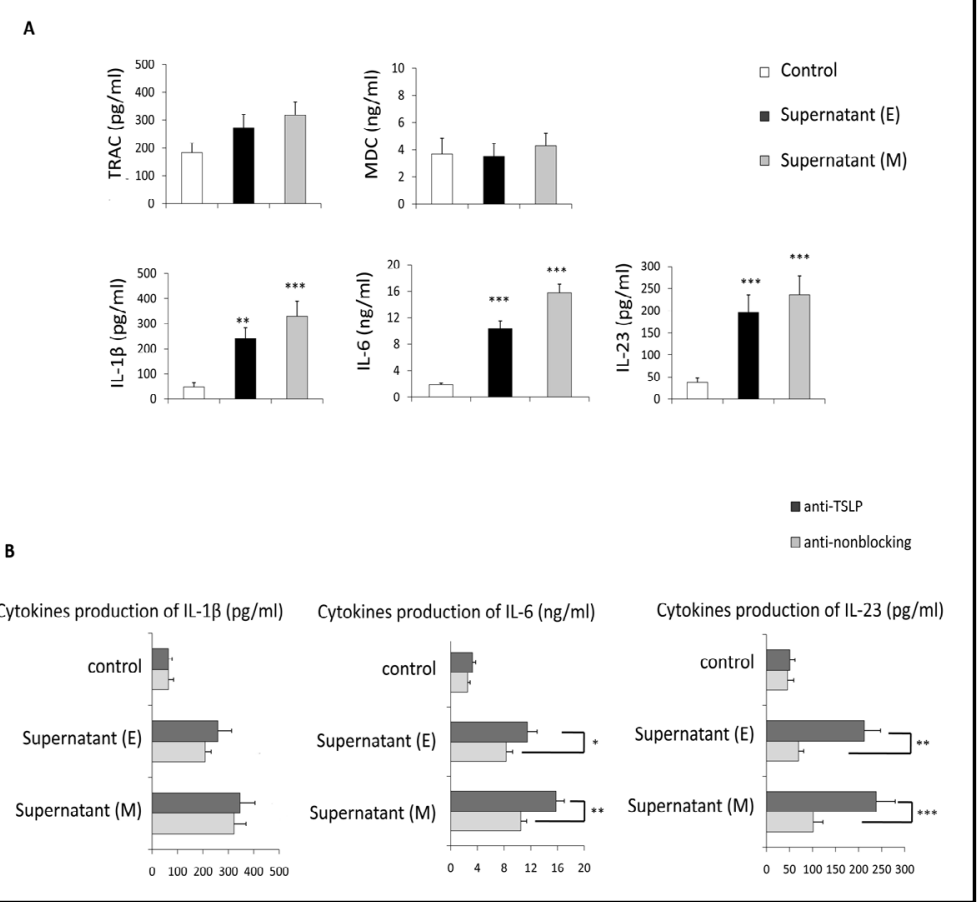

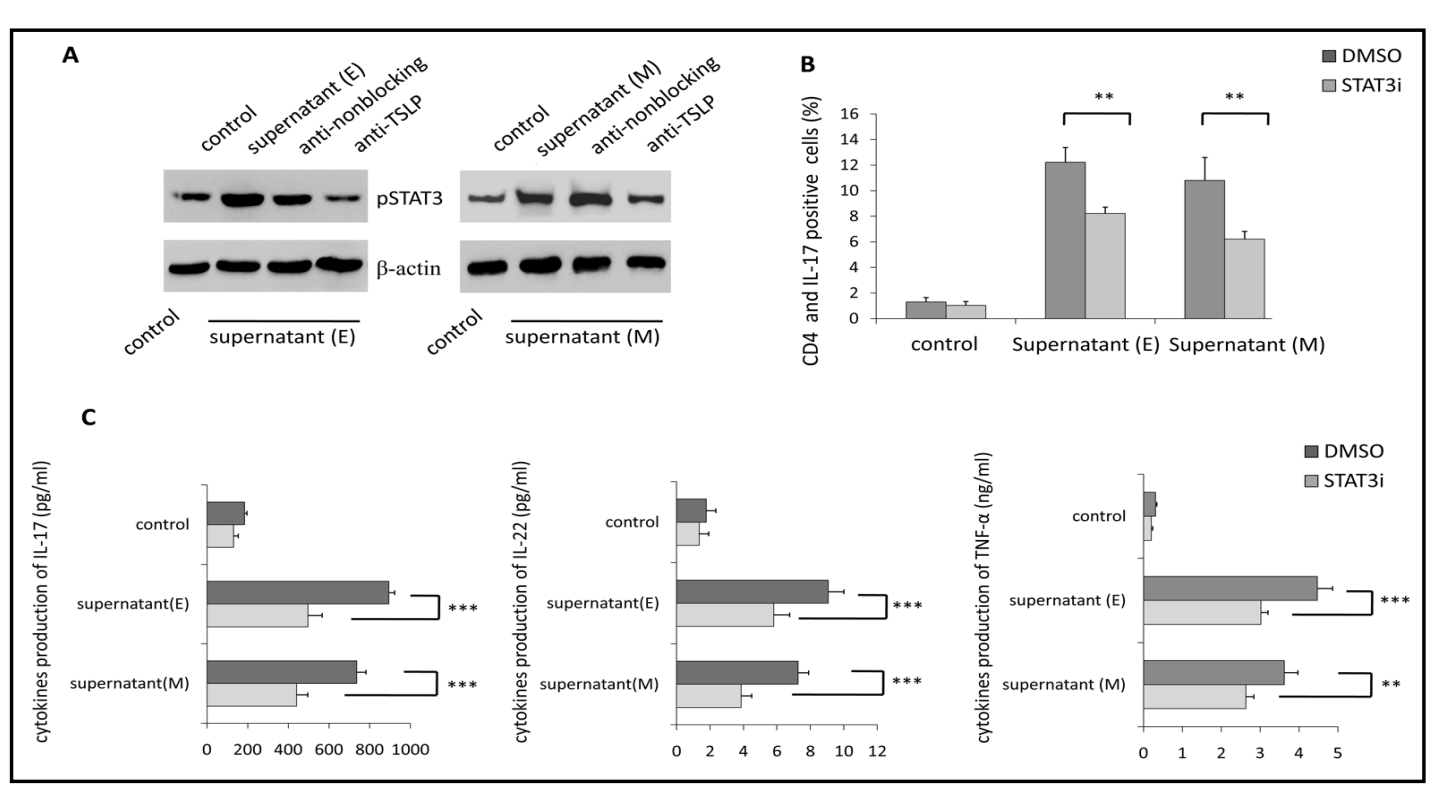

Fig. 8. STAT3 involved in TSLP induced Th17 inflammation in CD4 ${ }^{+} \mathrm{T}$ cells. DCs and CD4 ${ }^{+} \mathrm{T}$ cells were cultured as described above. $40 \mu \mathrm{mol} / \mathrm{l}$ STAT3 pathway inhibitor D4071 (Sigma, USA) or DMSO were added to the culture supernatants at the start of co-cultures. (A) Western blot analysis of the phosphorylation of STAT3 was measured in CD4+ $\mathrm{T}$ cells. (B) Frequencies of CD4+IL-17+ cells were analyzed statistically by SPSS 13.0. * indicated vs control, ${ }^{* *} \mathrm{p}<0.01$. (C) Cytokines of IL-17, IL-22 and TNF- $\alpha$ in supernatant were measured by ELISA. * indicated vs cells cultured with DMSO, ${ }^{* *} \mathrm{p}<0.01 ;{ }^{* * *} \mathrm{p}<0.001$. Data are presented as mean \pm SEM of at least three independent experiments.

supernatant TSLP treated DCs were next measured by ELISA. Figure 7A showed that while supernatant TSLP did not change production of Th2-cell attracting chemokines (TARC and 
MDC), supernatant from ox-LDL treated HUVECs or HVSMCs induced production of various inflammatory cytokines, including IL-1 $\beta$, IL-6, and IL-23 in DCs.

Our results also showed that anti-TSLP neutralizing antibody exhibited a strong reverse on supernatant induced production of IL-23 and IL-6 (Fig. 7B), but not IL-1 $\beta$ (Fig. 7B), suggesting that IL- 6 and IL-23 production in DCs may be attributed to ox-LDL induced TSLP in the supernatant.

\section{STAT3 signal pathway involved in TSLP-DCs induced Th17 inflammation}

It is recently reported that STAT3 is critical for commitment to the Th17 Lineage. To define whether STAT3 involved in TSLP-DCs induced Th17 inflammation, TSLP-DCs were co-cultured with CD4+ cells in presence or absence of human STAT3 pathway inhibitor (STAT3i) , and then Th17 differentiation and Th17 cytokines were measured. .

Figure 8A showed that while supernatant from HUVECs and HVSMCs induced STAT3 phosphorylation in human CD4+ cells, neutralizing antibody of TSLP could prevent STAT3 phosphorylation, suggesting that STAT3 pathway was involved in TSLP mediatedTh17 inflammation. Figure 8B showed that while DMSO had no effect on TSLP induced Th17 differentiation, STAT3 inhibitor partially inhibited TSLP induced Th17 differentiation. Figure 8C showed that STAT3 inhibitor prevent production of Th17 cytokines IL-17, IL-22 and TNF- $\alpha$, demonstrating that STST3 was critical for commitment to the Th17 lineage.

\section{Discussion}

$\mathrm{T}$ helper 17 (Th17) cells have recently been identified as an independent $\mathrm{T}$ cell subset, which characterized by the production of IL-17, IL-6, TNF- $\alpha$ and IL-22 [15]. Th17mediated inflammation has been confirmed to link with many autoimmune diseases, such as rheumatoid arthritis (RA), human psoriasis and inflammatory bowel diseases $[8,16]$. Although atherosclerosis is characterized by chronic dysregulated Th1 inflammation in the lesion, there are some evidences that Th17 inflammation also involved in atherosclerosis $[14,17,18]$. The frequency of Th17 cells and Th17 related cytokines are increased in local atherosclerotic lesion as well as in the peripheral circulation of patients with atherosclerosis, including patients with carotid artery plaques and acute coronary syndrome [14,18]. In animal models, while promoting atherosclerosis associated with increased Th17 inflammation, blockade of interleukin-17 A results in reduced atherosclerosis in apolipoprotein E-deficient mice $[13,18]$. Now, numerous studies on IL-7 or other Th17-related cytokines in animals or other evidence have shown that Th17 mediated inflammation is generally neither bystanders nor a consequence of plaque development, but instead directly promotes atherosclerosis.

TSLP is generally recognized as "switch for Th2 inflammation"[19]. It has been found highly expressed in atopic dermatitis and asthma, which characterized by Th2 dominant inflammation $[6,11]$. Our study demonstrated that while normal human vessels did not express TSLP, human atherosclerotic lesion exhibited a highly up-regulated TSLP expression. Atherosclerosis is not a Th2-drivening inflammation, but a Th1 and Th17-dominant inflammation- this contradictory trigger us to further elucidate the possible role of TSLP in atherosclerosis [1].

TSLP can be induced by epithelial cells, fibroblasts, keratinocytes and ECs in response to microbes, TLRs ligands and pro-inflammation cytokines, which is regulated by NF$\kappa B$ signaling $[20,21]$. Our previous study found that TSLP could also be inductive by angiotensin II in rat vascular smooth muscle cells and dependent on an NF- $\kappa B$ manner[10]. However, there are some differences in the details of TSLP regulation in different cell types. In normal human bronchial epithelial cells, TNF- $\alpha$ and IL- $1-\beta$, but not LPS, stimulated the expression of TSLP [22]. In primary human small airway epithelial cells, a cocktail of TNF- $\alpha$ and IL-1- $\beta$, peptidoglycan or poly (I-C), but not LPS, stimulated the production of TSLP [23]. Thus, TSLP regulation by pro-inflammatory cytokines or TLR ligands appears to differ in various cell types, depending on receptor expression or the presence of other cytokines. 
Lin et al.: A Potential Role of Thymic Stromal Lymphopoietin in Th17 Differentiation in Atherosclerosis

Normal human umbilical vein endothelial cells (HUVECs) and human vascular smooth muscle cell (HVSMCs) have not been reported to express TSLP. Consistent with these results, we did not found any expression of TSLP in normal HUVECs and HVSMCs. In contrast, TSLP was strongly up-regulated in these two types of cells after stimulated with ox-LDL, the major auto-antigen for pathogenesis of atherosclerosis, implying that TSLP over-expressed in lesion could be due to ox-LDL in the lesions.

Works from several groups suggest that the choice to activate T cell lineage developmental program is influenced, to a large extent, by the type of stimuli eliciting the response, antigendose, as well as, the strength of T cell-antigen-presenting-cell (APC) interactions. Dendritic cells, as a key linkage of innate and adaptive immunity, are the most professional APCs which can sense danger signals and prime naïve $\mathrm{T}$ cell immune response. The current paradigm for the role of TSLP in asthma and atopic dermatitis is that it activates DCs to polarize naive $\mathrm{CD}^{+} \mathrm{T}$ cells into a Th2 inflammation [6]. However, TSLP-DCs in our study did not induce a significant increase in Th2 related cytokines. In contrast, TSLP-DCs in our study could differentiate Th17 Cells and elevate Th17-associated cytokines secretion, including TNF- $\alpha$, IL-22 and IL-17. These data was partially consistent with recent study that TSLP and its receptor can enhance Th17-driven arthritis and tissue destruction in mouse models of arthritic, affirming one important role of TSLP is to induce Th17 mediated inflammation.

It is reported that TSLP activates DCs to strongly up-regulate the expression of costimulatory molecules, such as OX40 ligand (OX40L), CD80, and CD86, and an interaction between OX40 OX40L and OX40 was required for TSLP induced Th2 inflammation [24]. TSLP induced DCs to produce Th2-attracting chemokines TARC and MDC were also contributed to TSLP induced Th2 inflammation. However, TSLP activated DCs were cultured with CD4 ${ }^{+}$T cells in transwell condition in our study, which excluded interaction mechanism involved in TSLP-DCs induced Th17 inflammation, and suggested a cytokine-dependent manner was employed in our study. For this reason, profile of cytokines and chemokines in TSLP primed DCs were assessed in our study. We did not detect a significant increase of TRAC and MDC in TSLP treat DCs, but detected a significant release of IL- 6 and IL-23 in primed DCs. This deviation may be attributed to differences in the stimuli. Recently studies reported that compared with TSLP alone, TLRs plus TSLP could activate DCs to secrete IL-23, but not TRAC and MDC, to induce Th17 mediated inflammation in primed T cells [7]. We and others had previously reported ox-LDL could act as TLRs ligands to induce NF- $\mathrm{KB}$ signaling pathway $[25,26]$. Thus, it is reasonable that the supernatant, which contained ox-LDL as well as oxLDL induced TSLP, did not induce a production of TRAC and MDC, but a strong secretion of IL-6 and IL-23.

Additionally, Th1 cytokines, IFN- $\gamma$ was also increased in primed T cells, and this increase could not be affected by anti-TSLP neutralizing antibody. Ox-LDL had been shown to activate DCs to induce IL-12, which could synergize with IL-18 for full induction of IFN- $\gamma[27,28]$. Thus, the increased IFN- $\gamma$ production could be a result of inducible IL-12 in DCs. This result indicated that TSLP induced by ox-LDL activated DCs did not down-regulated Th1 cytokines in primed T cells, suggesting TSLP was more complicated when different stimuli and cell types were involved.

It is reported that while IL-6 was required for many stimuli, such as group A streptococcus, to induce Th17 inflammation, IL-23 was essential for the survival and stabilization of the Th17 phenotype [29, 30]. Our results showed that both IL-6 and IL-23 were involved in oxLDL induced TSLP-DCs differentiated Th17 inflammation. It is speculated that both IL- 6 and IL-23 may involved in ox-LDL induced TSLP-DCs differentiated Th17 inflammation.

Signal transducer and activator of transcription 3 (STAT3) is a cytoplasmic protein that functions as a transcriptional activator and plays a pivotal role in the regulation of different types of immune and inflammatory responses [31,32]. STAT3 protein exists in a latent form in the cytoplasm and gets phosphorylated at tyrosine residues upon receptor activation by cytokines such as IL-6. Once phosphorylated, STAT3 undergoes conformational change, dimerizes, and translocates to the nucleus where it binds the specific DNA motifs and activates the transcription of distinct groups of genes. Although STAT3 pathway has 
been vastly reported in commitment to the Th17 lineage [32], it is not clear whether STAT3 pathway involved in ox-LDL induced TSLP-DCs mediated Th17 inflammation. Our study showed that STAT3 was phosphorylated in TSLP-DCs treated T cells, but was diminished in the presence of anti-TSLP neutralizing antibody, suggesting STAT3 was implicated TSLPDCs mediated Th17 inflammation. This concept was further supported by STAT3 inhibitor, which could partially reversed TSLP-DCs mediated Th17 cell differentiation and Th17 cytokines production. Additionally, further experiments to elucidate whether other STAT3 independent pathways/mechanisms involved in TSLP-DCs mediated Th17 inflammation should be performed and guaranteed.

In conclusion, this study provides evidence to firstly demonstrate that ox-LDL could stimulate HVSMCs and HUECs to express TSLP. TSLP, in turn, could activate DCs to differentiate Th17 inflammation in naive $\mathrm{CD} 4^{+} \mathrm{T}$ cells. Considering the over-expression of TSLP in atherosclerotic lesion and the activation of Th17 inflammation in atherosclerosis, it is speculated that one role of TSLP in atherosclerosis may be was promoting Th17 inflammation.

\section{Acknowledgements}

This work was supported by National Natural Science Foundation of China to Dr. Dazhu Li (NO. 81170258). All of authors take responsibility for all aspects of the reliability and freedom from bias of the data presented and their discussed interpretation.

\section{References}

1 Libby P: Inflammation in atherosclerosis. Arterioscler Thromb Vasc Biol 2012;32:2045-2051.

$\checkmark 2$ Liu Z, Lu F, Pan H, Zhao Y, Wang S, Sun S, Li J, Hu X, Wang L: Correlation of peripheral th17 cells and th17associated cytokines to the severity of carotid artery plaque and its clinical implication. Atherosclerosis 2012;221:232-241.

3 Watanabe N, Hanabuchi S, Soumelis V, Yuan W, Ho S, de Waal Malefyt R, Liu YJ: Human thymic stromal lymphopoietin promotes dendritic cell-mediated CD4+ T cell homeostatic expansion. Nat Immunol 2004;5:426-434.

4 Blagoev B, Nielsen MM, Angrist M, Chakravarti A, Pandey A: Cloning of rat thymic stromal lymphopoietin receptor (tslpr) and characterization of genomic structure of murine tslpr gene. Gene 2002;284:161-168. Liu YJ: Tslp in epithelial cell and dendritic cell cross talk. Adv Immunol 2009;101:1-25.

6 Jariwala SP, Abrams E, Benson A, Fodeman J, Zheng T: The role of thymic stromal lymphopoietin in the immunopathogenesis of atopic dermatitis. Clin Exp Allergy 2011;41:1515-1520.

7 Tanaka J, Watanabe N, Kido M, Saga K, Akamatsu T, Nishio A, Chiba T: Human tslp and tlr3 ligands promote differentiation of th17 cells with a central memory phenotype under th2-polarizing conditions. Clin Exp Allergy 2009;39:89-100.

-8 Hartgring SAY, Willis CR, Dean CE, Broere F, van Eden W, Bijlsma JWJ, Lafeber FPJG, van Roon JAG: Critical proinflammatory role of thymic stromal lymphopoietin and its receptor in experimental autoimmune arthritis. Arthritis Rheum 2011;63:1878-1887.

-9 Lee HC, Sung SS, Krueger PD, Jo YA, Rosen HR, Ziegler SF, Hahn YS: Hepatitis C virus promotes Th17 responses through TSLP production by infected hepatocytes. Hepatology 2012. doi: 10.1002/hep.26128. [Epub ahead of print].

10 Zhao H, Li M, Wang L, Su Y, Fang H, Lin J, Mohabeer N, Li D: Angiotensin ii induces tslp via an at1 receptor/ nf-kappab pathway, promoting th17 differentiation. Cell Physiol Biochem 2012;30:1383-1397.

-11 Ying S, O'Connor B, Ratoff J, Meng Q, Fang C, Cousins D, Zhang G, Gu S, Gao Z, Shamji B, Edwards MJ, Lee $\mathrm{TH}$, Corrigan CJ: Expression and cellular provenance of thymic stromal lymphopoietin and chemokines in patients with severe asthma and chronic obstructive pulmonary disease. J Immunol 2008;181:2790-2798.

12 Lin J, Li M, Wang Z, He S, Ma X, Li D: The role of CD4+CD25+ regulatory t cells in macrophage-derived foamcell formation. J Lipid Res 2010;51:1208-1217. 
Lin et al.: A Potential Role of Thymic Stromal Lymphopoietin in Th17 Differentiation in Atherosclerosis

13 Gao Q Jiang Y, Ma T, Zhu F, Gao F, Zhang P, Guo C, Wang Q, Wang X, Ma C, Zhang Y, Chen W, Zhang L: A critical function of th17 proinflammatory cells in the development of atherosclerotic plaque in mice. J Immunol 2010;185:5820-5827.

14 Liu Z, Lu F, Pan H, Zhao Y, Wang S, Sun S, Li J, Hu X, Wang L: Correlation of peripheral th17 cells and th17associated cytokines to the severity of carotid artery plaque and its clinical implication. Atherosclerosis 2012;221:232-241.

$\checkmark 15$ Weaver CT, Hatton RD, Mangan PR, Harrington LE: Il-17 family cytokines and the expanding diversity of effector t cell lineages. Annu Rev Immunol 2007;25:821-852.

16 Chabaud M, Durand JM, Buchs N, Fossiez F, Page G, Frappart L, Miossec P: Human interleukin-17: A t cellderived proinflammatory cytokine produced by the rheumatoid synovium. Arthritis Rheum 1999;42:963970.

17 Xie JJ, Wang J, Tang TT, Chen J, Gao XL, Yuan J, Zhou ZH, Liao MY, Yao R, Yu X, Wang D, Cheng Y, Liao YH, Cheng X: The th17/treg functional imbalance during atherogenesis in apoe(-/-) mice. Cytokine 2010;49:185-193.

18 Smith E, Prasad KM, Butcher M, Dobrian A, Kolls JK, Ley K, Galkina E: Blockade of interleukin-17a results in reduced atherosclerosis in apolipoprotein e-deficient mice. Circulation 2010;121:1746-1755.

19 Liu YJ: Thymic stromal lymphopoietin: Master switch for allergic inflammation. J Exp Med 2006;203:269273.

20 He R, Geha RS: Thymic stromal lymphopoietin. Ann N Y Acad Sci 2010;1183:13-24.

-21 Soumelis V, Reche PA, Kanzler H, Yuan W, Edward G, Homey B, Gilliet M, Ho S, Antonenko S, Lauerma A, Smith K, Gorman D, Zurawski S, Abrams J, Menon S, McClanahan T, de Waal-Malefyt Rd R, Bazan F, Kastelein RA, Liu YJ: Human epithelial cells trigger dendritic cell mediated allergic inflammation by producing tslp. Nat Immunol 2002;3:673-680.

-22 Haro H, Komori H, Okawa A, Murakami S, Muneta T, Shinomiya K: Sequential dynamics of monocyte chemotactic protein-1 expression in herniated nucleus pulposus resorption. J Orthop Res 1997;15:734741.

23 Kanamaru Y, Sumiyoshi K, Ushio H, Ogawa H, Okumura K, Nakao A: Smad3 deficiency in mast cells provides efficient host protection against acute septic peritonitis. J Immunol 2005;174:4193-4197.

24 Gilliet M, Soumelis V, Watanabe N, Hanabuchi S, Antonenko S, de Waal-Malefyt R, Liu YJ: Human dendritic cells activated by tslp and cd40l induce proallergic cytotoxic t cells. J Exp Med 2003;197:1059-1063.

25 Li M, Lin J, Wang Z, He S, Ma X, Li D: Oxidized low-density lipoprotein-induced proinflammatory cytokine response in macrophages are suppressed by CD4CD25(+)Foxp3(+) regulatory t cells through downregulating toll like receptor 2-mediated activation of nf-kappab. Cell Physiol Biochem 2010;25:649656.

26 Su X, Ao L, Shi Y, Johnson TR, Fullerton DA, Meng X: Oxidized low density lipoprotein induces bone morphogenetic protein-2 in coronary artery endothelial cells via toll-like receptors 2 and 4 . J Biol Chem 2011;286:12213-12220.

27 Perrin-Cocon L, Coutant F, Agaugue S, Deforges S, Andre P, Lotteau V: Oxidized low-density lipoprotein promotes mature dendritic cell transition from differentiating monocyte. J Immunol 2001;167:3785-3791.

28 Fagundes CT, Costa VV, Cisalpino D, Amaral FA, Souza PR, Souza RS, Ryffel B, Vieira LQ, Silva TA, Atrasheuskaya A, Ignatyev G, Sousa LP, Souza DG, Teixeira MM: Ifn-gamma production depends on il-12 and il-18 combined action and mediates host resistance to dengue virus infection in a nitric oxide-dependent manner. PLoS Negl Trop Dis 2011;5:e1449.

29 Aggarwal S, Ghilardi N, Xie MH, de Sauvage FJ, Gurney AL: Interleukin-23 promotes a distinct CD4 T cell activation state characterized by the production of interleukin-17. J Biol Chem 2003;278:1910-1914.

-30 Dileepan T, Linehan JL, Moon JJ, Pepper M, Jenkins MK, Cleary PP: Robust antigen specific th17 t cell response to group a streptococcus is dependent on il-6 and intranasal route of infection. PLoS Pathog 2011;7:e1002252.

31 Levy DE, Darnell JE Jr: Stats: Transcriptional control and biological impact. Nat Rev Mol Cell Biol 2002;3:651-662.

-32 Egwuagu CE: Stat3 in CD4+ T helper cell differentiation and inflammatory diseases. Cytokine 2009;47:149156. 